\title{
Childhood-Onset Systemic Lupus Erythematosus in Southwestern Iran: A Clinical and Serological Study
}

\author{
Soheila ALYASIN, ${ }^{1,2}$ Reza AMIN,,$^{1,2}$ Mozhgan MOGHTADERI, ${ }^{1}$ Sara KASHEF, $, 1,2$ \\ ${ }^{1}$ Allergy Research Center, ${ }^{2}$ Department of Pediatrics, Shiraz University of Medical Sciences, Shiraz, Iran
}

\begin{abstract}
Objectives: This study aims to describe clinical manifestations of systemic lupus erythematosus (SLE) and laboratory assessment in children in the Southwestern Iran.

Patients and methods: This descriptive study included 32 patients ( 23 females, 9 males; mean age 10.5 years; range 3 to 16 years) with SLE who were referred to Rheumatology Outpatient clinic at Shiraz University of Medical Sciences between July 2011 and July 2012. A complete history of each patient was taken and the medical records were reviewed for clinical symptoms and laboratory test results.

Results: Twenty-three patients were newly diagnosed with SLE during the study. The most commonly involved organs were skin in 30 , joints in 21 , kidney in 18 and central nervous system in 10 patients. Laboratory findings at the time of diagnosis showed anemia in 19 , leukopenia in 15 and thrombocytopenia in three patients. Increased erythrocyte sedimentation rate and C-reactive protein values were detected in 27 patients and 16 patients, respectively. There was high level of double-stranded DNA in 30 and anti-nuclear antibodies in 27 patients. A total of four patients died within the study period, one from pulmonary hemorrhage, two from end-stage renal disease and the other one due to subdural hematoma and renal failure.

Conclusion: Our study results demonstrated that the clinical manifestations of SLE in childhood were mostly consistent with the previous reports of Iran and nearby Arabian countries. Kidney involvement was lower in our study; however with a higher number of patients with antinuclear antibody negative.

Keywords: Child; clinical manifestation; systemic lupus erythematosus.
\end{abstract}

Systemic lupus erythematosus (SLE) is an autoimmune disease with multi-organ involvement with a higher prevalence among young adult women. ${ }^{1,2}$ Even though SLE is usually rare in individuals less than five years of age, its prevalence is 10 to $15 \%$ during childhood. ${ }^{3}$ Children with SLE present with constitutional symptoms such as fever, fatigue, anorexia and lymphadenopathy. The disease affects several organs; however, the most commonly involved organs are the skin, joints, kidney, lung, central nervous system, blood vessels and blood cells. ${ }^{4}$ Of note, kidney involvement is present in up to $80 \%$ of children and is more severe than that seen in adults. ${ }^{5,6}$

Although underlying etiology of SLE is unknown, several factors including genetics, hormonal milieu and environmental exposures may increase the risk and affect severity and expression of the disease..$^{7-10}$

There are limited reports of childhood SLE in the Iranian population, in which several ethnic groups live. This study aims to describe clinical manifestations of SLE and laboratory assessment in children in the Southwestern Iran.

\section{PATIENTS AND METHODS}

This descriptive study included 60 children with suspected SLE who were referred to Rheumatology Outpatient clinic at Namazee Hospital affiliated to Shiraz University of Medical Sciences between July 2011 and July 2012. The diagnosis was based

Received: June 23, 2013 Accepted: August 18, 2013

Correspondence: Mozhgan Moghtaderi, M.D. Allergy Research Center, Shiraz University of Medical Sciences, Namazee Hospital, 71345-11351 Shiraz, Iran.

Tel: +98 7116474298 e-mail: moghtadery@sums.ac.ir

○2014 Turkish League Against Rheumatism. All rights reserved. 
on the American College of Rheumatology 1982 revised criteria for the classification of SLE. ${ }^{11}$

Twenty-eight children with drug-induced lupus, discoid lupus, other connective tissue diseases and those older than 18 years of age were excluded. A total of 32 children (23 females, 9 males; mean age 10.5 years; range 3 to 16 years) with lupus were enrolled. The study protocol was approved by the Institutional Ethics Committee and informed consents were obtained from the parents of the pediatric patients.

Demographic data and the family history of lupus in first-degree relatives were recorded for each patient by interview. Information about constitutional symptoms (fever, fatigue, anorexia and lymphadenopathy), skin, musculoskeletal system, kidney, central nervous system, lung and gastrointestinal tract in the course of disease were collected by medical chart.

Laboratory parameters including cell blood count, kidney function tests, erythrocyte sedimentation rate (ESR), C-reactive protein, urinalysis, antinuclear antibody (ANA), doublestranded DNA (dsDNA), Coombs' test, C3 and C4 complement levels were recorded using medical records. ANA and dsDNA levels were measured using the enzyme-linked immunosorbent assay method. Based on the result of urinalysis, a renal biopsy was performed by a pediatric nephrologist in patients with new-onset hematuria and/or proteinuria or the results of renal biopsy were noted. Each biopsy result was classified according to the World Health Organization (WHO) classification (class I normal, class II pure mesangial alteration, class III focal segmental glomerulonephritis, class IV diffuse glomerulonephritis, class $\mathrm{V}$ diffuse membranous glomerulonephritis, and class VI advanced sclerosing glomerulonephritis). ${ }^{12}$

Clinical and laboratory characteristics were presented as number and percentile.

\section{RESULTS}

All children were from the Southwestern Iran where all grew up. The female to male ratio was 2.5:1. The number of patients younger than five years was three (9\%). Table 1 shows the clinical manifestations of childhood-onset lupus in our patients. Newly diagnosed SLE children were 23 (71.9\%) during the study.
The most frequent hematological finding was anemia in 19 patients, of which 13 were Coombs' positive. A positive dsDNA was present in 30 patients (93.8\%) with SLE. Table 2 shows the laboratory results in children with SLE.

Eighteen of 32 patients (56\%) had hematuria and/or proteinuria, all of whom underwent renal biopsy. The most common feature of the renal biopsy was class IV WHO lupus nephritis $(n=8)$, followed by class II $(n=6)$, class $V(n=2)$, class I and class III ( $\mathrm{n}=1$ for each).

There was a positive history of SLE in firstdegree relatives of six patients (18.8\%). Four patients died during the study period, one from pulmonary hemorrhage, two later from endstage renal disease and the other from subdural hematoma and renal failure. No mortality was seen in the new cases of lupus.

\section{DISCUSSION}

The mean age at diagnosis of childhood SLE has varied among ethnic groups. Moreover, the mean age of 10.5 years in the present study is similar to what was found in Caucasians. As awareness of the occurrence of SLE in children increased, this study found three children younger than five years.

In this study, involvement with mucocutaneous manifestations was common. The same result was reported in SLE patients from other parts of Iran and neighboring Arab countries. ${ }^{13-15}$

Musculoskeletal involvement was seen in 65\% of our patients; this is less than that of other Iranian children (>75\%). ${ }^{13}$ As Southwestern Iran is located near Arab countries, the observed frequency was consistent with the Bahrain study. ${ }^{16}$

In addition, renal involvement in children is more common and more severe compared to adults. Kidney was the third most involved organ in our patients with an incidence of 56\%. Renal involvement was more frequent among children with SLE in other parts of Iran (64\%), Saudi Arabia (65\%), and Bahrain (81\%). ${ }^{13-15}$ However, longitudinal studies may be needed to follow possible abnormalities in kidney function of our patients in the future.

Renal biopsy should be performed in each patient with SLE, as there is a lack of a clear 


\begin{tabular}{|c|c|c|}
\hline \multirow[t]{2}{*}{ Clinical manifestations } & \multicolumn{2}{|c|}{ Present at any time } \\
\hline & $\mathrm{n}$ & $\%$ \\
\hline Constitutional & 30 & 93.8 \\
\hline Fever & 28 & 87.5 \\
\hline Fatigue & 19 & 59.4 \\
\hline Anorexia & 19 & 59.4 \\
\hline Lymphadenopathy & 2 & 6.3 \\
\hline Skin manifestation & 30 & 93.8 \\
\hline Malar rash & 20 & 62.5 \\
\hline Photosensitivity & 10 & 31.3 \\
\hline Alopecia & 3 & 9.4 \\
\hline Palmar erythema & 3 & 9.4 \\
\hline Maculopapular rash & 3 & 9.4 \\
\hline Oral ulcer & 2 & 6.3 \\
\hline Musculoskeletal & 21 & 65.6 \\
\hline Arthritis & 16 & 50.0 \\
\hline Arthralgia & 5 & 15.6 \\
\hline Myalgia & 2 & 6.3 \\
\hline Renal & 18 & 56.3 \\
\hline Isolated hematuria & 3 & 9.4 \\
\hline Proteinuria & 2 & 6.3 \\
\hline Hematuria and proteinuria & 14 & 43.8 \\
\hline Renal failure & 6 & 18.8 \\
\hline Neurologic & 10 & 31.3 \\
\hline Depression & 5 & 15.6 \\
\hline Seizure & 4 & 12.5 \\
\hline Cranial nerve palsy & 1 & 3.1 \\
\hline Pulmonary & 4 & 12.5 \\
\hline Plural effusion & 3 & 9.4 \\
\hline Pulmonary hemorrhage & 1 & 3.1 \\
\hline Gastroenterology & 5 & 15.6 \\
\hline Hepatosplenomegaly & 5 & 15.6 \\
\hline
\end{tabular}

correlation between clinical symptoms and renal involvement. Renal biopsy was performed in 18 children (56\%) in whom class IV lupus nephritis was the most dominant feature. A retrospective study on 60 prepubertal Iranian patients with lupus nephritis showed class IV nephritis to be the most frequent histological finding similar to our study. ${ }^{16}$ One reason could be that renal biopsies were often performed in severe suspected lupus cases.

In this study, neurological involvement was manifested by depression, seizure and cranial nerve palsy in $31 \%$ of patients. The lower incidence of neurological involvement may be explained by the lack of sufficient knowledge on systematic neurological examination in our children. Moreover, this result is similar to the report from Iran, Saudi Arabia and Bahrain. ${ }^{13-15}$

Pulmonary disease was seen in four patients (13\%). As pulmonary lupus sometimes has a poor outcome; one patient died from pulmonary hemorrhage shortly after the diagnosis. Anemia
Table 2. Laboratory findings in 32 children with systemic lupus erythematosus

\begin{tabular}{lcc}
\hline Laboratory findings & $\mathrm{n}$ & $\%$ \\
\hline Hemoglobin <8 g/Dl & 19 & 59.4 \\
Anemia with positive coombs & 13 & 40.6 \\
Anemia with negative coombs & 6 & 18.8 \\
Thrombocytopenia & 3 & 9.4 \\
Leukopenia & 15 & 46.9 \\
High ESR & 27 & 84.3 \\
High C-reactive protein & 16 & 50.0 \\
Positive ANA & 27 & 84.3 \\
Anti-dsDNA & 30 & 93.8 \\
Low C3 & 25 & 78.1 \\
Low C4 & 10 & 31.3 \\
\hline ESR: Erythrocyte sedimentation rate; ANA: Antinuclear antibody; Anti-dsDNA: \\
Anti-double-stranded deoxyribonucleic acid; C3: Complement component-3. \\
\hline
\end{tabular}

occurred in approximately one-half of our children with lupus; indicating an autoimmune hemolysis, which was detected by Coombs' test or it could be typical of chronic disease. Three patients suffered from thrombocytopenia, a result that was significantly lower than the statistics from other study groups. ${ }^{17,18}$

The most significant serological finding was a high ESR in $84 \%$ of our patients, as ESR often increases in case of an active disease.

Antibody to dsDNA is virtually pathognomonic for diagnosing SLE. High positivity of dsDNA $(94 \%)$ in our children suggests that this test is very useful in identifying childhood-onset lupus patients.

A negative ANA was detected in $16 \%$ of the participants. This finding was significantly higher than in previously published reports (2-3\%). ${ }^{19,20}$ An increased number of ANA negative patients can be explained by the technical inaccuracy in laboratories, too low levels of ANA in patient's serum, and by binding ANA in the form of immune complex. ${ }^{20}$ The serum level of complements are found to be decreased in active disease, and is often improved with treatment in SLE. Twenty percent of the SLE patients were of families in which one first-degree relative had lupus, probably due to the high consanguinity in the Iranian population.

In conclusion, our study findings demonstrated that the clinical manifestations of SLE in childhood were mostly consistent with the previous reports of Iran and nearby Arabian countries. Kidney involvement was lower in our study; however with a higher number of patients with ANA negative. 


\section{Declaration of conflicting interests}

The authors declared no conflicts of interest with respect to the authorship and/or publication of this article.

\section{Funding}

The authors received no financial support for the research and/or authorship of this article.

\section{REFERENCES}

1. Klein-Gitelman M, Reiff A, Silverman ED. Systemic lupus erythematosus in childhood. Rheum Dis Clin North Am 2002;28:561-77.

2. Nazarinia MA, Ghaffarpasand F, Shamsdin A, Karimi AA, Abbasi N, Amiri A. Systemic lupus erythematosus in the Fars Province of Iran. Lupus 2008;17:221-7.

3. Glidden RS, Mantzouranis EC, Borel Y. Systemic lupus erythematosus in childhood: clinical manifestations and improved survival in fifty-five patients. Clin Immunol Immunopathol 1983;29:196-210.

4. Putterman C, Caricchio R, Davidson A, Perlman H. Systemic lupus erythematosus. Clin Dev Immunol 2012;2012:437282.

5. Gloor JM. Lupus nephritis in children. Lupus 1998;7:639-43.

6. Wallace DJ, Hahn BH, Klippel JH. Clinical and laboratory features of lupus nephritis. In: Wallace DJ, Hahn BH, editors. Dubois' lupus erythematosus. 6th ed. Philadelphia: Lippincott Williams \& Wilkins; 2002. p. 1077-91.

7. Guerra SG, Vyse TJ, Cunninghame Graham DS. The genetics of lupus: a functional perspective. Arthritis Res Ther 2012;14:211.

8. Zandman-Goddard G, Solomon M, Rosman Z, Peeva E, Shoenfeld Y. Environment and lupus-related diseases. Lupus 2012;21:241-50.

9. Hiraki LT, Benseler SM, Tyrrell PN, Harvey E, Hebert D, Silverman ED. Ethnic differences in pediatric systemic lupus erythematosus. J Rheumatol
2009;36:2539-46.

10. Johnson AE, Gordon C, Palmer RG, Bacon PA. The prevalence and incidence of systemic lupus erythematosus in Birmingham, England. Relationship to ethnicity and country of birth. Arthritis Rheum 1995;38:551-8.

11. Tan EM, Cohen AS, Fries JF, Masi AT, McShane DJ, Rothfield NF, et al. The 1982 revised criteria for the classification of systemic lupus erythematosus. Arthritis Rheum 1982;25:1271-7.

12. Weening JJ, D'Agati VD, Schwartz MM, Seshan SV, Alpers CE, Appel GB, et al. The classification of glomerulonephritis in systemic lupus erythematosus revisited. J Am Soc Nephrol 2004;15:241-50.

13. Moradinejad MH, Zamani GR, Kiani AR, Esfahani T. Clinical features of juvenile lupus erythematosus in Iranian children. Acta Reumatol Port 2008;33:63-7.

14. Bahabri S, Sabban EA, Al Rashed A, Al-Mayouf S, Al Mazyed A, Abdulrazik A, et al. Juvenile systemic lupus erythematosus in 60 Saudi children. Ann Saudi Med 1997; 17:612-5.

15. Al-Mosawi Z, Al-Hermi BE, Al-Saad KK, Farid EM, Makki HA. Juvenile systemic lupus erythematosus in Bahrain. A tertiary referral center experience. Saudi Med J 2009;30:667-72.

16. Taheri S, Beiraghdar F. Lupus nephritis in Iranian children: a review of 60 patients. Ren Fail 2011;33:499-505.

17. Domiciano DS, Shinjo SK. Autoimmune hemolytic anemia in systemic lupus erythematosus: association with thrombocytopenia. Clin Rheumatol 2010;29:1427-31.

18. Mashhadi MA, Bari Z. Thrombotic thrombocytopenic purpura and deep vein thrombosis as the presenting manifestations of systemic lupus erythematosus: A case report and review of literature. J Res Med Sci 2011;16:1082-8.

19. Chaubey VK, Chhabra L. Cold agglutinin-induced haemolysis in association with antinuclear antibodynegative SLE. BMJ Case Rep 2013 Jun 10;2013.

20. Kim HA, Chung JW, Park HJ, Joe DY, Yim HE, Park $\mathrm{HS}$, et al. An antinuclear antibody-negative patient with lupus nephritis. Korean J Intern Med 2009;24:76-9. 